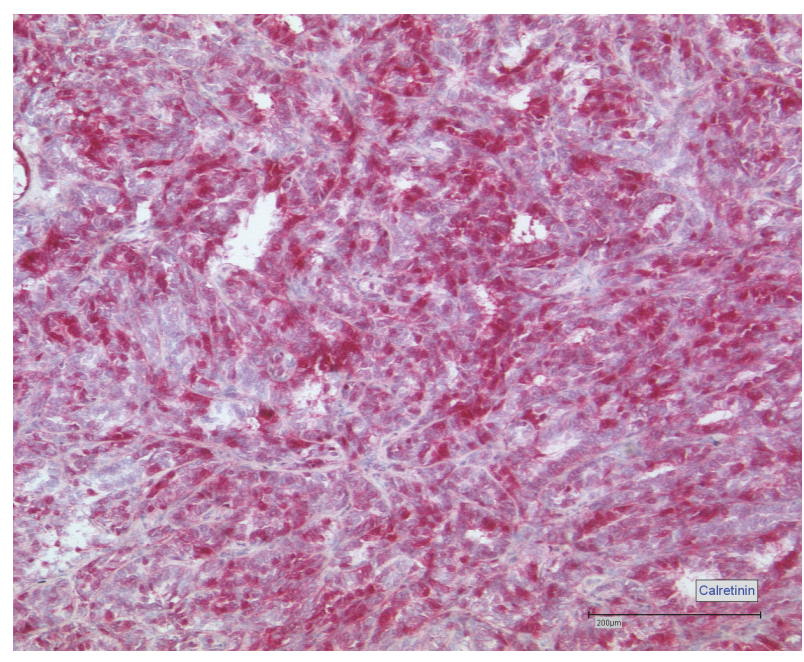

Abstract 1099 Figure 1

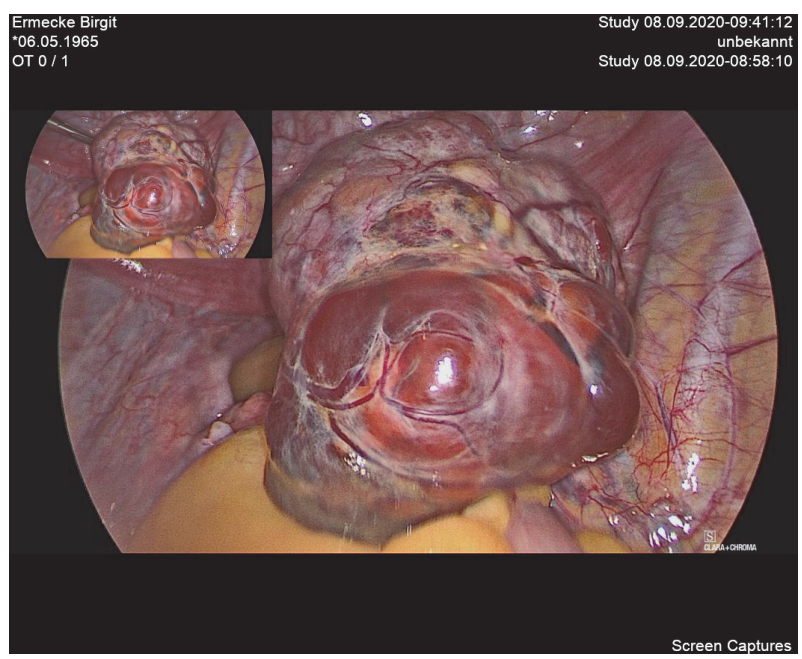

Abstract 1099 Figure 2

patient was prepared for a laparoscopic bilateral salpingooophorectomy which was successfully performed. Intraoperatively, an approx. $6.5 \times 4 \mathrm{~cm}$ twisted, round, solid-cystic structure with an irregular surface was discovered on the right fallopian tube. The mass was excised laparoscopically with both of adnexa using electrocautery. The postoperative phase was uneventful. The histology initially described an undifferentiated tumor which was shown by immunohistochemistry analysis to be a Wolffian tumor.

Result(s)* After negative staging with abdomen and thorax CT, the case was presented to the hospital's tumor board where treatments were discussed and a total laparoscopic hysterectomy was indicated. The patient underwent a total laparoscopic hysterectomy and abdominal biopsies with negative histology.

The rarity of the disease has led to poorly-defined therapeutic options. It has been considered that the most effective therapy is complete surgical resection with hysterectomy and bilateral adnexectomy. ${ }^{8,9}$ Sole tumor resection is a risk factor for relapse. The role of adjuvant chemotherapy or radiation is controversial. $^{8}$
Conclusion* Due to rarity of cases and data concerning the malignant progression of such tumors, more studies are required to decide upon the appropriate management. Although some cases are benign, there has been evidence of malignant behavior. The efficacy of adjuvant therapy is still in question. Following surgical treatment, regular follow-up examinations should be planned for the long-term.

\section{CARCINOSARCOMA OF THE FALLOPIAN TUBE: A CASE REPORT AND REVIEW OF THE LITERATURE}

${ }^{1}$ ME Capilna* , ${ }^{2} \mathrm{AL}$ Cozlea. "'George Emil Palade' University of Medicine, Pharmacy, Science and Technology, First Obstetrics and Gynecology Clinic, Târgu Mureş, Romania; ' 'George Emil Palade' University of Medicine, Pharmacy, Science and Technology, First Obstetrics and Gynecology Clinic, Târgu Mureş, Romania

\subsection{6/ijgc-2021-ESG0.290}

Introduction/Background* Carcinosarcoma, also known as Malignant Mixed Müllerian Tumor (MMMT), includes both malignant mesenchymal and epithelial elements. The fallopian tube is the most uncommon localization of this pathology, being associated with poor prognosis and an extremely aggressive progression.

Methodology A case of a 65-year-old postmenopausal patient with a final histological diagnosis of fallopian MMMT staged FIGO IC2, synchronous with a serous endometrial intraepithelial carcinoma, is described. From the literature, 99 previous case reports were reviewed. Gathered data was statistically analyzed together with the case from our clinic's experience,

Result(s)* Age between 41 and 60 years old, symptoms at presentation and CT/RMN tumor evidence could be prognosis factors $(\mathrm{P}<0.05)$. Omentectomy $(\mathrm{OR}=0.3545)$ and pelvic lymphadenectomy $(\mathrm{OR}=0.3732)$ are significant factors for survival $(\mathrm{P}<0.05)$. Fimbrial localization of tumor could be a negative prognosis factor ( $\mathrm{OR}=4.263)$, as well as heterologous type of tumor $(\mathrm{OR}=2.880)$. Chemotherapy improves survival $(\mathrm{OR}=0.2679)$ while radiotherapy has no influence on the prognosis.

Conclusion* Reporting this rare histology could be important to obtain more data regarding the optimal oncologic management, aiming to improve patients'survival.

\section{COMPLICATIONS RATES IN GYNECOLOGIC ONCOLOGY. A SINGLE CENTER EXPERIENCE}

DE Vlachos*, N Thomakos, V Pergialiotis, C Theofanakis, V Theodoulidis, D Haidopoulos, A Rodolakis. National and Kapodistrian University of Athens, 1st Department of Ob/Gyn, Athens, Greece

\subsection{6/ijgc-2021-ESG0.291}

Introduction/Background* Surgery in gynecological cancer remains one of the primary modalities of treatment. The procedures are now standardized and online video libraries are a source of knowledge and training for young surgeons. This accumulation of knowledge has promoted the surgical skills and increased the radicality of the procedures, especially in debulking surgeries that are now extended in upper abdomen or even the thoracic cavity. This increased radicality inevitable increases the complications during and after surgery. 